\title{
Elevated soluble TNFa levels and upregulated TNFa mRNA expression in purified peripheral blood monocyte subsets associated with high-grade hepatocellular carcinoma
}

\author{
C. Martín-Sierra ${ }^{1,2,3+}$ (D) R. Martins ${ }^{4,5,6,7 \dagger}$, M. Coucelo ${ }^{8}$, A. M. Abrantes ${ }^{6,7}$, R. C. Oliveira ${ }^{6,7,9}$, J. G. Tralhão $0^{4,5,6,7}$, \\ M. F. Botelho ${ }^{6,7}$, E. Furtado ${ }^{4}$, M. R. Domingues ${ }^{10}$, A. Paiva ${ }^{1,2,3,11^{*}}$ (D) and P. Laranjeira ${ }^{1,2,3}$
}

\begin{abstract}
Background: Chronic inflammation is involved in the initiation and progression of various cancers, including liver cancer. The current study focuses on the characterization of the peripheral immune response in hepatocellular carcinoma (HCC) and cholangiocarcinoma (CCA) patients, before and after surgical procedure, in order to assess the effect of tumor resection in the immune system homeostasis and to determine possible prognostic factors associated with high-grade tumors. We developed a whole-blood assay to monitor immune alterations and functional competence of peripheral monocytes in a group of 10 healthy individuals (HG), in $20 \mathrm{HCC}$ patients and 8 CCA patients, by multi-color flow cytometry, qRT-PCR, and ELISA techniques.

Results: The GRT-PCR analysis showed an upregulation of TNFa expression by classical and intermediate monocytes purified from HCC patients presenting tumors in grade G3-G4 as compared to G1-G2 HCC patients. Moreover, ELISA assay confirmed elevated serum levels of TNFa in G3-G4 compared to G1-G2 HCC patients. A significant decrease of circulating non-classical monocytes was detected in both CCA and HCC patients before and after surgical procedure. In addition, a functional defect in circulating classical and intermediate monocytes was observed in both groups of cancer patients when compared to the $\mathrm{HG}$, with partial recovery after the surgical intervention.

(Continued on next page)
\end{abstract}

\footnotetext{
*Correspondence: artur.paiva@chuc.min-saude.pt

${ }^{+} \mathrm{C}$. Martín-Sierra and R. Martins contributed equally to this work.

${ }^{1}$ Flow Cytometry Unit, Clinical Pathology Service, Centro Hospitalar e

Universitário de Coimbra, Praceta Prof. Mota Pinto, Ed. S. Jerónimo, $3^{\circ}$ piso,

Coimbra, Portugal

${ }^{2}$ Coimbra Institute for Clinical and Biomedical Research (iCBR), Faculty of

Medicine, University of Coimbra, Coimbra, Portugal

Full list of author information is available at the end of the article
}

(c) The Author(s). 2020 Open Access This article is licensed under a Creative Commons Attribution 4.0 International License, which permits use, sharing, adaptation, distribution and reproduction in any medium or format, as long as you give appropriate credit to the original author(s) and the source, provide a link to the Creative Commons licence, and indicate if changes were made. The images or other third party material in this article are included in the article's Creative Commons licence, unless indicated otherwise in a credit line to the material. If material is not included in the article's Creative Commons licence and your intended use is not permitted by statutory regulation or exceeds the permitted use, you will need to obtain permission directly from the copyright holder. To view a copy of this licence, visit http://creativecommons.org/licenses/by/4.0/ The Creative Commons Public Domain Dedication waiver (http://creativecommons.org/publicdomain/zero/1.0/) applies to the data made available in this article, unless otherwise stated in a credit line to the data. 


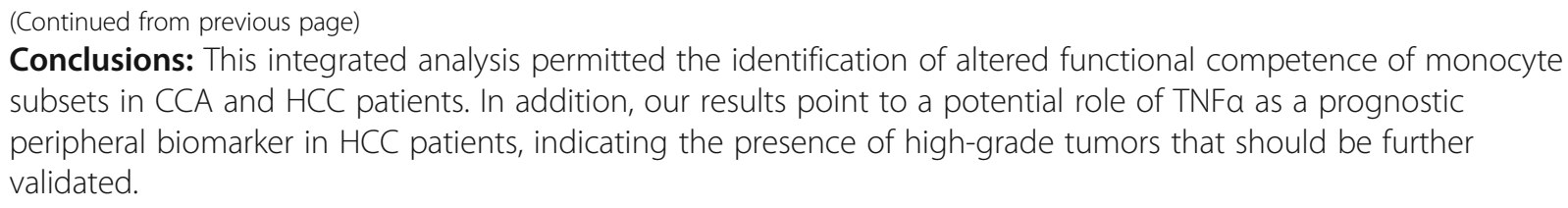

Keywords: Flow cytometry, qRT-PCR, Hepatocellular carcinoma, Cholangiocarcinoma, Circulating monocytes, TNFa

\section{Background}

Liver cancer is the second most common cause of cancer-related death worldwide, with a steady increasing incidence and mortality [1] and, therefore, a major public health challenge. It is considered an immunogenic cancer because $90 \%$ of cases develop under conditions of chronic inflammation $[1,2]$. This inflammation conducts to the development of tumors and it is associated with higher tumor immunogenicity. For this reason, the most suitable therapeutic strategies to be applied in these types of carcinomas would be immunotherapeutic approaches [3]. Hepatocellular carcinoma (HCC) is the most frequent liver cancer and is associated to high morbidity and mortality rates $[1,4]$. It presents poor prognosis, generally due to its late presentation and thus, late diagnostic. Cholangiocarcinoma (CCA), a malignancy that originates from biliary epithelia, is an aggressive cancer with a high mortality rate [5] and, along with HCC, represents a major primary liver cancer [2]. CCA is difficult to diagnose due to its silent and nonspecific clinical features and, in most cases, the symptoms occur when the tumor has reached an advanced stage [6]. For patients with advanced disease, there are only limited therapeutic treatment options that provide limited benefits for a subset of patients. Therefore, novel therapeutic options are needed [7]. Partial surgical resection and liver transplantation are potentially curative treatments in selected patients with HCC and CCA $[8,9]$. Unfortunately, a high postoperative tumor recurrence rate significantly decreases long-term survival outcomes [9].

Prognosis of cancer patients is based on tumor-related factors as well as on host-related factors, including systemic immune cell activation [10]. Given the difficulties in acquiring liver tissues, circulating biomarkers as well as comprehensive studies monitoring the peripheral immune system homeostasis, are needed. In fact, it has been demonstrated that immune monitoring of peripheral blood (PB) cells might lead to the identification of biomarkers, which could serve to predict prognosis and/ or therapy response [11]. Due to its immunogenic origin, further knowledge of the changes on immune cell populations may help define the potential of the combination of therapies, such as adoptive immunotherapy and/or checkpoint inhibition [12]. Moreover, monitoring of the peripheral immune response after surgical resection will provide information to advance our understanding of the mechanisms underlying the clinical response to surgical resection.

In the present study, we have characterized, by multicolor flow cytometry, the frequency, composition and activity of circulating monocyte subsets in $\mathrm{PB}$ samples from HCC and CCA patients, in order to understand the immune status of these patients, and to obtain evidence about the changes in the proportion or functions of these populations, for application in the clinical diagnosis and future development of treatments to HCC and CCA.

\section{Results}

Alterations of peripheral blood leukocyte subsets in CCA and HCC patients

From the data obtained by flow cytometry immunophenotyping, CCA and HCC patients displayed a decrease in the frequency and absolute numbers of $\mathrm{PB}$ monocytes, both at T0 and T1, compared to the HG. Regarding monocyte subsets, CCA patients displayed a significant decrease in the absolute numbers of PB classical, intermediate and non-classical monocytes, both at T0 and T1, when compared to the HG, and a significant decrease in the relative frequency of non-classical monocytes (within circulating monocytes) at T0 in comparison to the HG, that was partially restored at T1. HCC patients presented a significant decrease in the relative frequency and absolute numbers of circulating nonclassical monocytes, both at $\mathrm{T} 0$ and $\mathrm{T} 1$, when compared to the HG, and a significant increase in the relative frequency of classical monocytes, both at T0 and T1, as compared to the HG. Interestingly, the relative frequency of intermediate monocytes presenting high-level surface expression of HLA-DR [13] $\left(\mathrm{HLADR}^{++}\right)$was significantly decreased in HCC patients at T0 in comparison to the HG (Table 1).

\section{Functional alterations of peripheral blood monocyte subsets in CCA and HCC patients}

The different leukocyte subpopulations were identified and distinguished based on CD45 positivity, their typical forward scatter (FSC) and side scatter (SSC) light 
Table 1 Frequency (\%) and absolute numbers (cells/ $\mu$ L) of peripheral blood monocyte subsets in cholangiocarcinoma (CCA) and hepatocellular carcinoma (HCC) patients, both at the time of surgical procedure (TO) and once the patients were recovered from surgery (T1), and in healthy individuals (HG). Frequencies of monocyte subsets are related to the total of monocytes

\begin{tabular}{|c|c|c|c|c|c|}
\hline & \multicolumn{2}{|l|}{$\begin{array}{l}\text { CCA } \\
\boldsymbol{N}=8\end{array}$} & \multicolumn{2}{|l|}{$\begin{array}{l}\mathrm{HCC} \\
\boldsymbol{N}=20\end{array}$} & \multirow[t]{2}{*}{$\begin{array}{l}\mathrm{HG} \\
\boldsymbol{N}=10\end{array}$} \\
\hline & T0 & $\mathrm{T} 1$ & T0 & $\mathrm{T} 1$ & \\
\hline \% Monocytes (in whole blood) & $2,4(1,0-6,6)^{a, b}$ & $4,9(2,2-7,0)^{a}$ & $4,2(1,5-10)^{a, b}$ & $5,5(2,7-9,5)^{a}$ & $8,2(5,7-12)$ \\
\hline Monocytes/ $\mu \mathrm{L}$ & $141(51-330)^{a}$ & $219(128-306)^{a}$ & $329(135-850)^{\mathrm{a}}$ & $353(127-733)$ & $503(285-757)$ \\
\hline$\%$ Classical & $89(76-98)$ & $84(75-92)$ & $89(41-97)^{a}$ & $90(74-96)^{a}$ & $83(70-88)$ \\
\hline Classical monocytes $/ \mu \mathrm{L}$ & $139(40-287)^{a, b}$ & $161(111-282)^{\mathrm{a}}$ & $318(129-1361)^{c}$ & $264(102-601)$ & $393(171-651)$ \\
\hline$\%$ Intermediate & $4,4(0,3-14)$ & $7,3(4,8-12)$ & $2,5(0,5-42)$ & $4,8(1,7-13)$ & $5,5(2,8-8,2)$ \\
\hline Intermediate monocytes/ $\mu \mathrm{L}$ & $6,1(0,4-36)^{a}$ & $15(8,3-17)^{a}$ & $12(0,8-194)$ & $13(5,8-79)$ & $23(17-51)$ \\
\hline$\% \mathrm{HLADR}^{+}$Intermediate & $32(23-83)$ & $44(33-63)$ & $45(16-72)^{a}$ & $38(23-80)$ & $33(13-56)$ \\
\hline$\% \mathrm{HLADR}^{++}$Intermediate & $68(17-77)$ & $56(37-67)$ & $55(28-84)^{a}$ & $62(20-77)$ & $68(44-87)$ \\
\hline$\%$ Non-Classical & $6,0(0,4-7,5)^{a}$ & $5,0(1,1-14)$ & $3,6(1,0-22)^{a}$ & $3,5(0,9-13)^{a}$ & $11(5,2-28)$ \\
\hline Non-classical monocytes/uL & $6,7(0,6-24)^{a}$ & $6,5(1,4-24)^{a}$ & $15(2,4-60)^{a}$ & $13(2,6-58)^{a}$ & $61(26-84)$ \\
\hline
\end{tabular}

Independent-samples Mann-Whitney $U$ test was performed to compare: each group of patients vs healthy group (a); CCA vs HCC (b), The Wilcoxon test was performed to compare T1 vs T0 (c), all of them with a significance level of $0.05(p<0.05)$. The results are given by median (minimum value-maximum value)

dispersion properties, and their positivity for specific membrane markers as shown in Fig. 1a. Regarding TNF $\alpha$ production after stimulation (Fig. $1 \mathrm{~b}$ and Supplementary Table S2), a statistically significant decrease in the frequency of TNF $\alpha$ producing classical monocytes, for CCA $(74 \% \pm 29)$ and $\mathrm{HCC}(85 \% \pm 20)$ at T0, was observed when compared to the HG $(98 \% \pm 2)$. HCC patients displayed a significant decrease in the frequency of $\mathrm{TNF} \alpha$ producing classical monocytes at $\mathrm{T} 1(90 \% \pm 16)$ as compared to the HG $(98 \% \pm 2)$, while no differences were found for CCA patients at T1. A statistically significant decrease in the frequency of TNF $\alpha$ producing intermediate monocytes with mid-level surface expression of HLA-DR (HLADR ${ }^{+}$) was observed for CCA patients $(87 \% \pm 20)$ vs. HG $(99 \% \pm 1)$. Additionally, both CCA $(93 \% \pm 10)$ and HCC $(97 \% \pm 5)$ patients displayed a statistically significant decrease in the frequency of TNF $\alpha$ producing intermediate monocytes with highlevel surface expression of HLA-DR $\left(\mathrm{HLADR}^{++}\right)$at T0 in comparison to the HG $(100 \% \pm 0)$.

We did not observe significant differences when comparing patients presenting tumors classified as G1-G2 to patients presenting tumors classified as G3-G4 (data not shown).

After the purification of monocyte subpopulations by cell sorting, the mRNA levels of CX3CR1 and TNF $\alpha$ were measured by qRT-PCR. The expression levels of CX3CR1 mRNA among classical monocytes were significantly decreased in both groups of cancer patients at T0 when compared to the HG, with partial recovery at T1 in both groups of cancer patients (Fig. 2a). A significant increase in the expression levels of CX3CR1 mRNA among intermediate monocytes was detected in HCC patients at T1. Moreover, the expression levels of
CX3CR1 mRNA by non-classical monocytes were significantly decreased in $\mathrm{HCC}$ patients at T0 when compared to the HG, with a recovery at T1. The same pattern was observed in CCA patients but without reaching statistical significance.

On the other hand, no differences were observed on TNF $\alpha$ mRNA expression among classical and nonclassical monocytes, in CCA or HCC patients, when compared to the HG. Nevertheless, CCA patients displayed a significant increase of TNF $\alpha$ mRNA in intermediate monocytes at T0 in comparison to the HG, while at T1, TNF $\alpha$ mRNA levels approach to those observed in HG (Fig. 2a).

Taking into account the histopathological grade of the tumors, HCC patients presenting tumors classified as grade G3-G4 displayed significantly higher levels of TNF $\alpha$ mRNA in classical and intermediate monocytes at T0 in comparison to HCC patients with G1-G2 tumors; non-classical monocytes displayed the same tendency, without reaching statistical significance (Fig. 2b). The same pattern was observed in CCA patients but without reaching statistical significance (Fig. 2b).

\section{Analysis of chemokines and cytokines serum levels in CCA and HCC patients}

With regard to the chemokines analyzed by ELISA, elevated serum levels of CCL20 were found in both CCA and HCC patients at T0, compared to the HG. While CCA patients showed a partial recovery at T1, HCC patients' CCL20 levels remained high at T1 (Fig. 3a). Circulating levels of CXCL10 were also increased in HCC patients, at $\mathrm{T} 0$, in comparison to the HG, followed by a partial recovery at T1. Conversely, no significant differences were observed for CCL2 among the studied groups (Fig. 3a). 

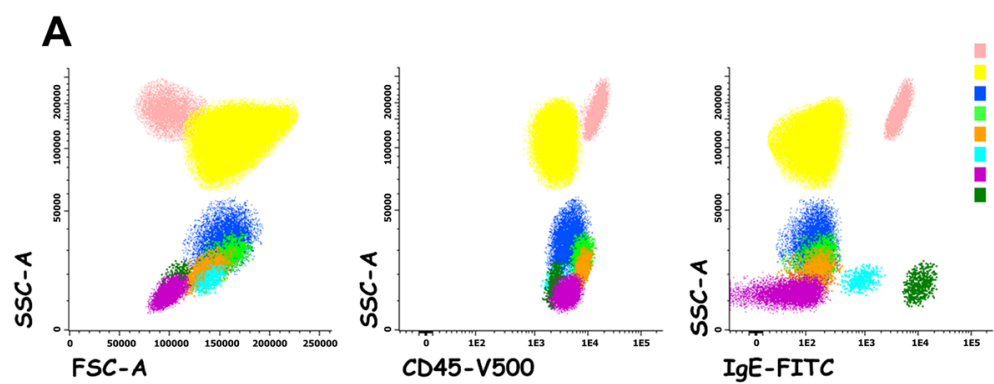

Eosinophils

Neutrophils

Classical monocytes

Intermediate monocytes
Non-classical monocytes

$\mathrm{mDCs}$

Lymphocytes

Basophils

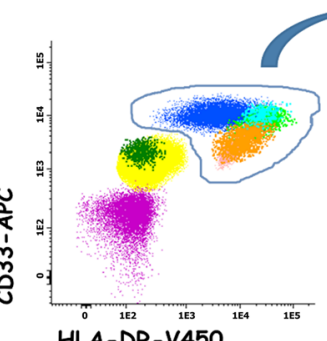

HLA-DR-V450
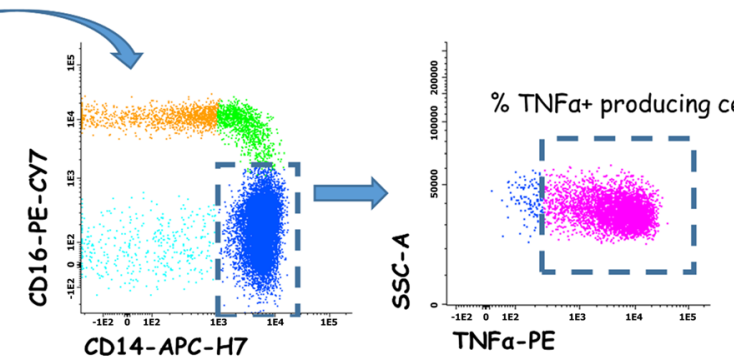

B

After stimulation with LPS + IFNY

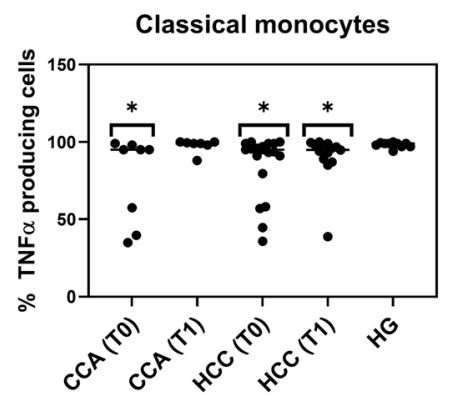

Intermediate monocytes HLADR+
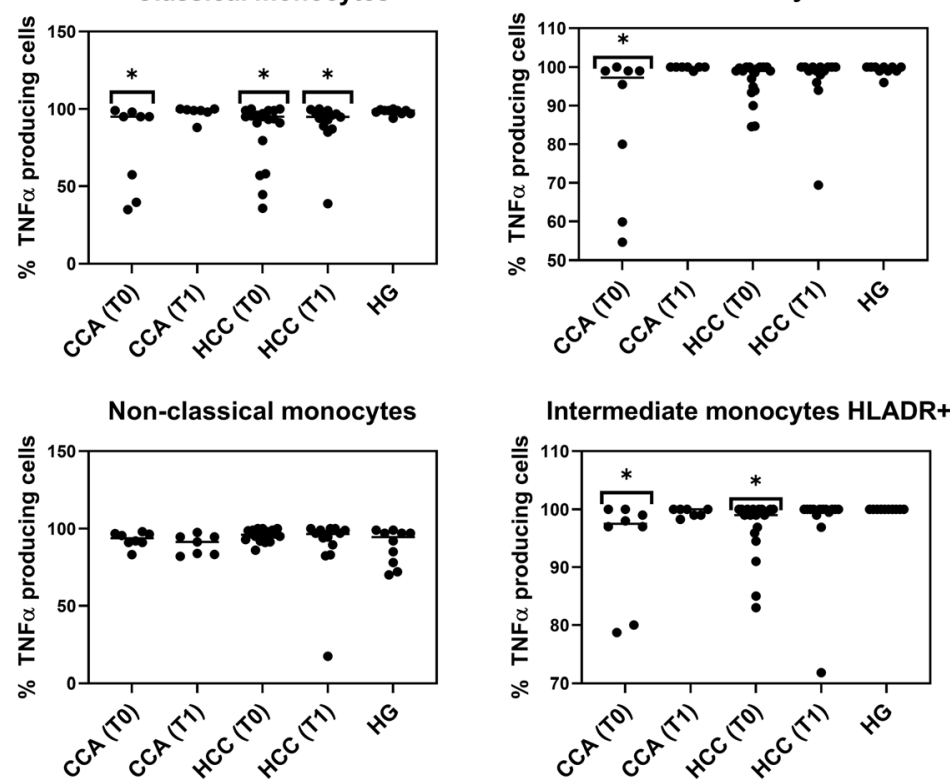

Fig. 1 Phenotypic and functional characterization of circulating monocyte subsets. a Bivariate dot plot histograms illustrating the identification of circulating leukocyte subsets: eosinophils (light pink events), neutrophils (yellow events), classical monocytes (blue events), intermediate monocytes (light green events), non-classical monocytes (orange events), myeloid dendritic cells (mDCs, light blue events), lymphocytes (purple events), and basophils (green events), and an example bivariate dot plot histogram illustrating TNFa production (indicated with dashed rectangle) by classical monocytes after stimulation with LPS and IFN- $\gamma$. b Dot plots with the frequency of classical monocytes, intermediate monocytes with mid-level surface expression of HLA-DR (HLADR ${ }^{+}$, intermediate monocytes presenting high-level surface expression of HLA-DR $\left(H L A D R^{++}\right)$and non-classical monocytes producing TNFa, after stimulation with LPS plus IFNy, in cholangiocarcinoma $(C C A, N=8)$ and hepatocellular carcinoma ( $H C C, N=20)$ patients, both at T0 and $T 1$, and in the healthy group ( $H G, N=10)$. Statistically significant differences were considered when $p<$ 0.05 ; ${ }^{*}$ between the groups indicated in the figure and the HG

Concerning serum levels of TNF $\alpha$, CCA patients displayed a significant increase of TNF $\alpha$ at $\mathrm{T} 0$, in comparison to the HG, partially restored at $\mathrm{T} 1$, while no significant differences were observed for HCC patients (Fig. 3a).
Notably, serum levels of TNFa were significantly higher in G3-G4 compared to G1-G2 HCC patients (at T0). A similar pattern was observed in CCA patients without reaching statistical significance (Fig. 3b). 


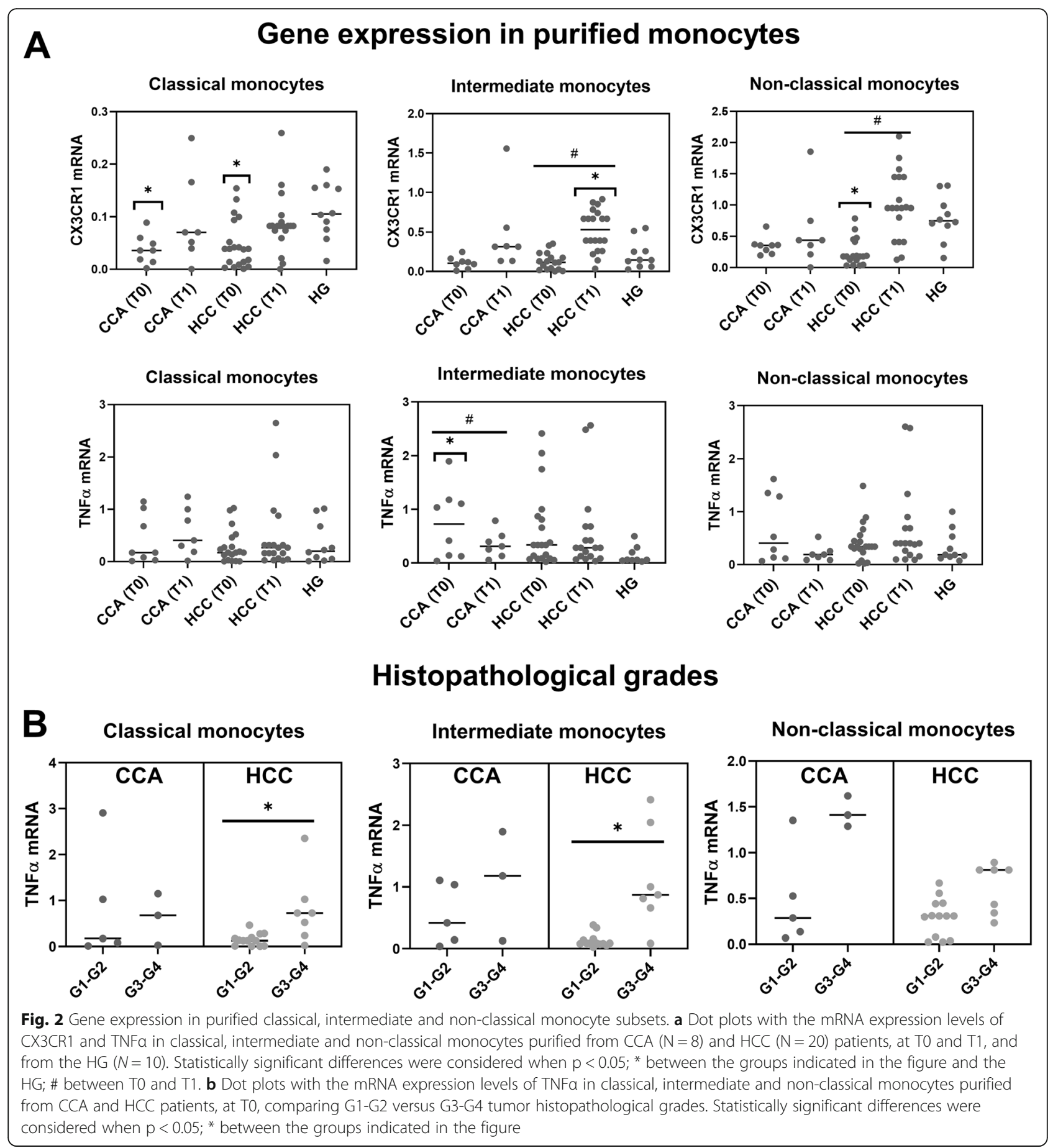

\section{Discussion}

The current study has identified a functional defect on some circulating monocyte subsets in HCC and CCA patients, regarding their capability to produce TNF $\alpha$ under stimulation, displaying a partial recovery after surgical procedure. Moreover, a peripheral proinflammatory state in HCC and CCA patients was observed, and, a potential role for TNF $\alpha$ circulating levels, as well as TNF $\alpha$ mRNA expression by classical and intermediate monocytes, as prognostic peripheral biomarkers in HCC patients, indicating the presence of high-grade (G3 or G4) tumors, was revealed.

Inflammatory factors affect nearly all the stages of tumor development and the effectiveness of the applied therapies. TNF $\alpha$ is considered one of the most important inflammatory mediators of the cancer-associated inflammatory networks. In this regard, preclinical studies in breast cancer have suggested that TNF $\alpha$ promotes 


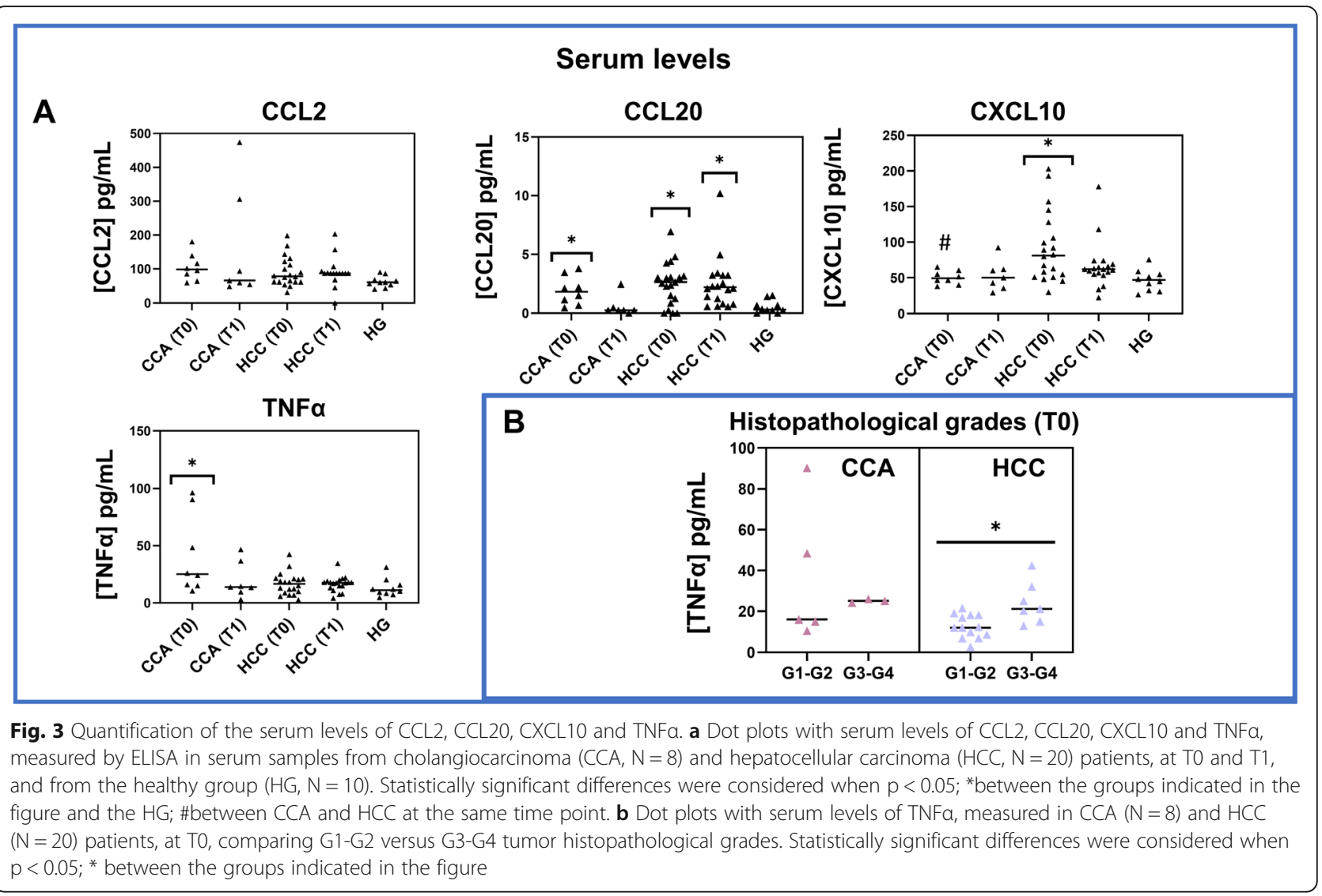

tumor growth in vivo and could be considered a therapeutic target [14]. In the present study, we pointed out an upregulation of TNFa mRNA expression in peripheral classical, intermediate and non-classical monocytes purified from HCC patients presenting tumors classified as grade G3-G4, as compared to G1-G2 HCC patients. Moreover, in line with these results, we detected a significant increase of TNF $\alpha$ serum levels in HCC patients with tumors in grade G3-G4 as compared to grade G1G2 HCC. In this regard, further studies will be needed to validate the potential use of TNF $\alpha$ serum levels as a prognostic factor or indicator of histopathological grading. Supporting this idea, it has been reported that aberrant elevated TNF $\alpha$ levels might promote not only tumor growth, but also invasion and poor prognosis [15]. Additionally, a previous work in a rat liver cancer model demonstrated that TNF $\alpha$ inhibition and deletion could decrease tumor incidence and showed that clinically TNF $\alpha$ expression was correlated to hepatic progenitor cells activation and HCC recurrences [16].

Regarding the characterization of circulating monocytes, we observed a significant decrease in the frequency and absolute counts of PB monocytes, both in CCA and HCC patients, at T0, as compared to the HG. Interestingly, values continued to be diminished comparatively to the HG after surgical procedure (at T1).
The observed decrease in the frequency of circulating monocytes could be associated to an increase in the frequencies of circulating neutrophils since previous works have identified elevated levels of circulating neutrophils that were significantly and independently associated with poorer prognosis in HCC [17-19]. Peripheral blood monocytes constantly enter the liver to replenish hepatic macrophages and dendritic cells, playing important roles in the pathogenesis of inflammatory disorders [20]. Our previous findings had already shown altered frequencies and altered absolute numbers of circulating $F c \varepsilon \mathrm{RI}^{+}$ monocytes and myeloid dendritic cells in CCA and HCC patients, as well as functional defects in both cell subsets [21]. Thus, we wondered whether other monocyte subsets might be affected in these patients. The results of the present study extend our previous findings, showing alterations on monocytes function, as well as a significant decrease in the relative frequency and absolute numbers of circulating non-classical monocytes both in CCA and HCC patients. These values remained diminished as compared to the HG at T1, excepting the frequency of non-classical monocytes in CCA patients, that was partially restored after tumor resection. Nonclassical monocytes "patrol" the vasculature via a mechanism that requires the fractalkine receptor CX3CR1, covering apoptotic endothelial cells and sensing danger 
signals coming from the tissue [22]. A previous work has shown that this population of monocytes reduces tumor metastasis by recruiting NK cells [23]. Therefore, we hypothesized that the observed decrease in this monocyte subset may be having a role in cancer progression. Additionally, it has been suggested that non-classical monocytes are crucial regulators in the pathogenesis of chronic liver disease in humans, by the secretion of abundant cytokines, perpetuating chronic inflammatory processes within the liver and by directly activating hepatic stellate cells that, in turn, can secrete multiple chemokines for monocyte recruitment into the liver [24]. Therefore, indicating that the modulation of monocytesubset recruitment into the liver may represent possible novel approaches for interventions targeting proinflammatory actions of monocyte subsets in liver cancer.

When evaluating monocyte function, we observed, at T0, a significant decline in TNF $\alpha$ production by classical and $\mathrm{HLADR}^{++}$intermediate monocyte subsets, under LPS plus IFN $\gamma$ stimulation, in both CCA and HCC patients as compared to the HG, whereas no significant differences were detected for non-classical monocytes. TNF $\alpha$ production by $\mathrm{HLADR}^{+}$intermediate monocyte was also affected in CCA patients before tumor resection, but not in HCC patients. The frequencies of TNF $\alpha$-producing cells seem to be restored after tumor resection, excepting classical monocyte subsets that displayed a significant decrease in TNF $\alpha$ production in HCC patients at T1. These data indicate a functional defect in classical and intermediate monocytes in both, CCA and HCC patients that it is partially restored for intermediate monocytes at $\mathrm{T} 1$. In line with these results, previous studies in other carcinomas have reported a diminished percentage of TNF $\alpha$-producing $\mathrm{CD} 14^{+}$cells in $\mathrm{PB}$ of lung adenocarcinoma patients, showing that malignant cells inhibited the capability of monocytes to produce TNF $\alpha$ [25]. Thus, the decreased TNF $\alpha$ expression, observed in classical and intermediate monocytes, may be caused by tumor cells, but also, may influence tumor progression and relapse, since an altered response of innate immune cells might underscore a reduced capacity to mount an efficient antitumor immune response [26]. Monocytes can differentiate into a variety of macrophage or dendritic cell subtypes that can either activate or inhibit the immune response [27], and it has been shown that CX3CR1 is rapidly downregulated during monocytes differentiation [28]. In relation with this process, classical and non-classical monocytes purified from CCA and HCC patients presented a downregulation of the mRNA expression of the fractalkine receptor, CX3CR1, at T0, when compared to the HG, with partial recovery at $\mathrm{T} 1$, indicating a possible differentiation process in these monocyte subsets occurring before surgical procedure. Additionally, CX3CR1 expression could participate in promoting cell-to-cell interactions with an inflamed endothelium, as well as increasing monocytes survival [28]. We also detected, in intermediate monocytes from CCA patients, an upregulation of TNF $\alpha$ mRNA expression at T0 that was restored after surgical procedure, which is further supported by the results obtained from the measurement of serum TNF $\alpha$ levels, in which we detected significantly elevated TNFa serum levels in CCA patients at T0, that were restored at T1. Indicating an important role of peripheral TNFo in CCA associated inflammatory response.

In addition, we detected elevated circulating levels of CXCL10 in HCC patients, before surgery, that were only partially restored at T1. CXCR3 is the receptor for CXCL10, a member of the CXC chemokine family with pro-inflammatory and anti-angiogenic properties [29] that has been associated with a variety of human diseases, including tumor development, metastasis, and dissemination. More importantly, CXCL10 has been identified as a major biological marker mediating disease severity and may be used as a prognostic indicator for various diseases [30], thus, its possible role as disease severity biomarker for liver cancers should be further evaluated. Moreover, circulating levels of CCL20 in HCC patients before and after tumor resection were significantly increased, in comparison to the HG, as well as in CCA patients at $\mathrm{T} 0$, which presented a partial recovery after tumor resection. CCL20, alternatively called liver activation regulated chemokine (LARC), is a strong chemoattractant for leukocytes expressing its sole receptor CCR6 [31]. Many studies have shown that the CCL20/CCR6 axis contribute to the initiation and progression of $\mathrm{HCC}$ by mediating the migration of circulating $\mathrm{T}$ cells into the tumor microenvironment [32]. Thus, the observed increase in the circulating levels of CCL20 indicates a probable migration of $\mathrm{T}$ cells to the tumor microenvironment of these carcinomas.

The detected increase in the circulating levels of CCL20, CXCL10, and TNFa suggests a peripheral proinflammatory state in HCC and CCA patients and an activate state for monocyte subsets although classical and intermediate monocyte subsets displayed a functional defect at T0 and, therefore, a limited capacity to respond under further stimulation processes.

\section{Conclusions}

This integrated analysis permitted the identification of different immune background in CCA and HCC patients, as well as altered functional competence of circulating monocytes in these carcinomas. Intriguingly, most of these alterations appear to be restored in CCA patients after tumor resection, however, most of them remained in $\mathrm{HCC}$ patients after surgical procedure. 
Moreover, this work has demonstrated that flow cytometry serves as a powerful analytical platform for the rapid characterization of individual cells within heterogenic cell populations and it may help in monitoring functional competence of immune cell populations to better evaluate immune dysfunctions in cancer patients. This approach could be applied in clinical routine to evaluate innate and adaptive immunity and to monitor responses to different treatments. In addition, the identified immune alterations should be further studied to consider clinically relevant therapeutic targets. A better understanding of the potential of inflammation and innate immunity to inhibit tumor progression should lead to the development of new and improved immunomodulatory approaches for the treatment of liver carcinomas. Notably, our results point out a potential role for $\mathrm{TNF} \alpha$ as a prognostic factor in HCC patients indicating the presence of high-grade tumors. However, further studies should be conducted with larger sample sizes to validate the findings we have presented and correlate these findings with post-surgery outcomes after long-term followup.

\section{Methods}

The aim of the present study was to characterize, by multi-color flow cytometry, the frequency, composition and activity of circulating monocyte subsets in $\mathrm{PB}$ samples from HCC and CCA patients, in order to understand the immune status of these patients, and to obtain evidence about the changes in the proportion or functions of these populations, for application in the clinical diagnosis and future development of treatments to HCC and CCA.

\section{Patients and healthy individuals}

PB samples were collected from 20 patients with HCC (3 women and 17 men; average age: $62.2 \pm 14.5$ years) and 8 patients with CCA (5 women and 3 men; average age: $61.0 \pm 14.7$ years), just before the beginning of the surgical resection (T0), and once the patient was completely recovered from the surgical procedure (T1). In addition, a group of 10 age- and sex-matched healthy individuals was included in the present study as a control group (HG). None of the patients included in this study took any medication before surgical procedure nor at T1. Nonetheless, 7 HCC patients took tacrolimus following liver transplantation which targets $\mathrm{T}$ lymphocytes $[33,34]$ but has no effect over monocyte function [35].

Patients were classified according to the 8th TNM classification and their clinical background is summarized in Table 2 .

We did not identify any significant difference between HCC patients presenting liver cirrhosis and HCC patients without liver cirrhosis for all the parameters analyzed in this study. Therefore, all HCC patients were included in the same group.

\section{In vitro stimulation of cytokine production by circulating monocytes}

To evaluate the TNF $\alpha$ production by classical, intermediate and non-classical monocytes, PB samples were collected from participants and healthy individuals into lithium heparin (Becton Dickinson Biosciences, BD, San Jose, CA, USA) and stimulated with lipopolysaccharide (LPS) plus interferon gamma (IFNY), as previously described in Martín-Sierra et al. 2019 [21]. Brefeldin-A was added to inhibit protein transport in Golgi complex, as it redistributes intracellularly produced proteins from the cis/medial Golgi complex to the endoplasmic reticulum. All samples were then incubated in a $5 \% \mathrm{CO}_{2}$ humid atmosphere, at $37^{\circ} \mathrm{C}$, for $6 \mathrm{~h}$.

Immunophenotypic analysis was performed by using a seven-color monoclonal antibodies (mAbs) combination, detailed in Supplementary Table S1 (tube 1). Samples were aliquoted $(300 \mu \mathrm{L})$ and stained with the mAbs for surface proteins antigens (CD45, HLA-DR, IgE, CD16, CD33 and CD14). After extracellular staining, we followed the protocol previously described in Martín-Sierra et al. 2019 [21].

\section{Flow cytometry data acquisition and analysis}

The stained samples were acquired in a FACSCanto II flow cytometer (BD) and the data were analyzed with Infinicyt 1.8 software (Cytognos SL, Salamanca, Spain).

For the identification of the distinct monocyte subsets, we used the following gating strategy: we selected the monocyte population by its characteristic FSC/SSC light dispersion properties together with high expression of CD45, HLA-DR, and CD33. Within monocytes, classical, intermediate and non-classical monocyte subpopulations were distinguished based mainly on the expression levels of CD16 and CD14. Classical monocytes express high levels of CD14 in the absence of CD16, together with high expression of CD33 and HLA-DR; intermediate monocytes express high levels of CD14 as well, but display an increasing expression of CD16, associated to a slight decrease of CD33 expression, compared to classical monocytes; in turn, non-classical monocytes show CD16 positivity with a decreasing expression of CD14, they present the highest expression of CD45 along with the lowest expression of CD33 among the three monocyte subpopulations (as displayed in Fig. 1a).

When these cell subsets were characterized and identified in the Infinicyt software, we plotted each population independently to determine TNF $\alpha$ production levels by each subset of monocytes (as indicated in the last plot of Fig. 1a). 
Table 2 Clinical data from hepatocellular carcinoma (HCC) and cholangiocarcinoma (CCA) patients enrolled in this study. Number of patients and frequencies (\%) are indicated

\begin{tabular}{llll}
\hline & & CCA & HCC \\
& Stage I & $1(13 \%)$ & $3(15 \%)$ \\
\hline TNM & Stage II & $4(50 \%)$ & $15(75 \%)$ \\
& Stage IIIA & $0(0 \%)$ & $1(5 \%)$ \\
& Stage IV & $3(38 \%)$ & $1(5 \%)$ \\
& G1 & $2(25 \%)$ & $2(10 \%)$ \\
Histologic grade (G) & G2 & $3(38 \%)$ & $11(55 \%)$ \\
& G3 & $3(38 \%)$ & $6(30 \%)$ \\
& G4 & $0(0 \%)$ & $1(5 \%)$ \\
HBsAg & Positive & $0(0 \%)$ & $1(5 \%)$ \\
HCV & Positive & $0(0 \%)$ & $6(30 \%)$ \\
Vascular microinvasion & Positive & - & $8(40 \%)$ \\
Cirrhosis & & $0(0 \%)$ & $15(75 \%)$ \\
Relapse & & $3(38 \%)$ & $1(5 \%)$ \\
Death & & $3(38 \%)$ & $2(10 \%)$ \\
Liver Transplant & & $0(0 \%)$ & $7(35 \%)$ \\
\hline
\end{tabular}

\section{Cell purification by fluorescence-activated cell sorting}

Classical $\left(\mathrm{CD} 14^{++} \mathrm{CD} 16^{-}\right)$, intermediate $\left(\mathrm{CD} 14^{++} \mathrm{CD} 16^{+}\right)$ and non-classical $\left(\mathrm{CD} 14^{+} \mathrm{CD} 16^{++}\right)$monocyte subpopulations from $\mathrm{PB}$ were purified by fluorescence-activated cell sorting (FACS), using a FACSAria III flow cytometer (BD) according to their typical phenotype. Thus, the four-color mAbs combination used (Supplementary Table S1, tube 2) allowed the identification of each monocyte subset.

For subsequent mRNA expression analysis, purified cell populations were prepared and stored as previously described in our laboratory [36].

\section{RNA isolation and quantitative real-time reverse transcriptase-polymerase chain reaction (qRT-PCR)}

We used the RNeasy ${ }^{\text {ma }}$ Micro Kit (Qiagen) according to the manufacturer's instructions to extract and purify total RNA content from each cell subpopulation. Reverse transcription and relative quantification of gene expression was performed as previously described in our laboratory [36], using optimized primers for TNF $\alpha$, CX3CR1 and endogenous control glyceraldehyde 3phosphate dehydrogenase (GAPDH) (Qiagen), according to the manufacturer's instructions.

\section{Assessment of cytokine and chemokine serum concentrations}

PB samples were collected from patients and healthy individuals into VACUETTE Serum Gel tubes (GreinerBio-One, Kremsmünster, Austria) and were centrifuged for $10 \mathrm{~min}$ at $2000 \mathrm{~g}$. Subsequently, serum samples were subdivided into small aliquots to be stored at $-80^{\circ} \mathrm{C}$ until tested for cytokine and chemokines levels. ELISA kits were used to determine monocyte chemotactic protein-1 (MCP-1 or CCL2, Thermo Scientific), MIP-3a (CCL20, Thermo Scientific), IP-10 (CXCL10, Thermo Scientific) and TNF $\alpha$ (Thermo Scientific) serum levels, according to the manufacturer's instructions.

\section{Statistical analysis}

Data are presented as the mean values with their standard deviation or as the median with the minimum and maximum values. The non-parametric Mann-Whitney and Kruskal-Wallis multiple comparison tests were employed, using the Statistical Package for Social Sciences software (SPSS, version 25, IBM, Armonk, NY, USA). Moreover, the non-parametric Wilcoxon signedrank test of non-independent data was performed to compare T1 vs T0. Statistical significance was considered when $p<0.05$. GraphPad Prism software was used to create the graphics.

\section{Supplementary information}

Supplementary information accompanies this paper at https://doi.org/10. 1186/s12950-020-00243-7.

Additional file 1: Table S1. Panel of $\mathrm{mAb}$ reagents (with clones and commercial source) used for the immunophenotypic characterization and for cell purification by fluorescence-activated cell sorting of tumor cells and immune cells. Table S2. Absolute numbers (cells/ $\mu \mathrm{L}$ ) and frequencies (\%) of TNFa producing peripheral blood monocyte subsets in cholangiocarcinoma (CCA) and hepatocellular carcinoma (HCC) patients, both at the time of surgical procedure (TO) and once the patients were recovered from surgery $(\mathrm{T} 1)$, and in healthy individuals (HG).

\section{Acknowledgements}

Not applicable.

\section{Authors' contributions}

CMS processed the samples, performed the cell sorting, the flow cytometry, the ELISA assays and the molecular biology analyses, analyzed the results and was a major contributor in the writing of the manuscript. RM, AMA, RCO, JGT, MFB and EF provided the biological samples, performed patients' selection, revised the clinical data and reviewed the manuscript. PL supervised the data analysis, help in sample processing and reviewed the manuscript. MC supervised the molecular biology analyses. MRD supervised the work and reviewed the manuscript. Finally, AP conceived the main idea of the work, interpreted the results and reviewed the manuscript. CMS was a PhD student in the Biochemistry program at University of Aveiro and this work is part of her doctoral thesis. All authors read and approved the final manuscript.

\section{Funding}

This work was supported by the European Commission's Horizon 2020 research and innovation programme for the Marie Sklodowska-Curie (grant agreement number 675132, MSCAITN-ETN MASSTRPLAN) to Centro Hospitalar e Universitário de Coimbra, Coimbra (Portugal) and by COMPETE 2020 Programa Operacional Competitividade e Internacionalização (POCI) [POCl01-0145-FEDER-007440, UID/NEU/04539/2013] to CNC.IBILI. 


\section{Availability of data and materials}

The authors declare that the main data supporting the results of the present study are available within the article and its Supplementary Information files. Extra data are available from the corresponding author upon request.

\section{Ethics approval and consent to participate}

The experimental protocols were approved by the Ethical Committee of the Faculty of Medicine, University of Coimbra, Coimbra, Portugal (CE-136/2016). All procedures performed involving human participants were in accordance with the ethical standards of Ethical Committee of the Faculty of Medicine, University of Coimbra, Coimbra, Portugal (CE-136/2016), and with the 1964 Helsinki declaration and its later amendments or comparable ethical standards.

\section{Consent for publication}

All participants gave their signed informed consent before entering in the study.

\section{Competing interests}

The authors declare that they have no competing interests.

\section{Author details}

${ }^{1}$ Flow Cytometry Unit, Clinical Pathology Service, Centro Hospitalar e Universitário de Coimbra, Praceta Prof. Mota Pinto, Ed. S. Jerónimo, $3^{\circ}$ piso, Coimbra, Portugal. ${ }^{2}$ Coimbra Institute for Clinical and Biomedical Research (iCBR), Faculty of Medicine, University of Coimbra, Coimbra, Portugal. ${ }^{3}$ Center for Innovative Biomedicine and Biotechnology (CIBB), University of Coimbra, Coimbra, Portugal. ${ }^{4}$ Unidade Transplantação Hepática Pediátrica e de Adultos, Centro Hospitalar e Universitário de Coimbra (UTHPA, CHUC), Coimbra, Portugal. ${ }^{5}$ Serviço de Cirurgia Geral, Unidade HBP, Centro Hospitalar e Universitário de Coimbra (CHUC), Coimbra, Portugal. " Instituto de Biofísica, IBILI, Faculdade de Medicina, Universidade de Coimbra, Coimbra, Portugal. ${ }^{7}$ Coimbra Institute for Clinical and Biomedical Research (iCBR) area of Environment Genetics and Oncobiology (CIMAGO), Faculty of Medicine, University of Coimbra, Coimbra, Portugal. ${ }^{8}$ Unidade de Hematologia Molecular, Serviço de Hematologia Clínica, Centro Hospitalar e Universitário de Coimbra (CHUC), Coimbra, Portugal. ' ${ }^{9}$ Serviço de Anatomia Patológica, Centro Hospitalar e Universitário de Coimbra (CHUC), Coimbra, Portugal. ${ }^{10}$ Mass Spectrometry Centre, Department of Chemistry \& QOPNA, University of Aveiro, Campus Universitário de Santiago, Aveiro, Portugal. ${ }^{11}$ Instituto Politécnico de Coimbra, ESTESC-Coimbra Health School, Ciências Biomédicas Laboratoriais, Coimbra, Portugal.

Received: 13 December 2019 Accepted: 13 March 2020 Published online: 30 March 2020

\section{References}

1. Llovet JM, Zucman-Rossi J, Pikarsky E, Sangro B, Schwartz M, Sherman M, Gores G. Hepatocellular carcinoma. Nat Rev Dis Prim. 2016. https://doi.org/ 10.1038/nrdp.2016.18.

2. Sia D, Villanueva A, Friedman SL, Llovet JM. Liver Cancer cell of origin, molecular class, and effects on patient prognosis. Gastroenterology. 2017. https://doi.org/10.1053/J.GASTRO.2016.11.048.

3. Pardee AD, Butterfield LH. Immunotherapy of hepatocellular carcinoma. Oncoimmunology. 2012. https://doi.org/10.4161/onci.1.1.18344.

4. Rahib L, Smith BD, Aizenberg R, Rosenzweig AB, Fleshman JM, Matrisian LM Projecting Cancer incidence and deaths to 2030: the unexpected burden of thyroid, liver, and pancreas cancers in the United States. Cancer Res. 2014. https://doi.org/10.1158/0008-5472.CAN-14-0155.

5. Ghouri YA, Mian I, Blechacz B. Cancer review: Cholangiocarcinoma. J Carcinog. 2015. https://doi.org/10.4103/1477-3163.151940.

6. Blechacz B, Komuta M, Roskams T, Gores GJ. Clinical diagnosis and staging of cholangiocarcinoma. Nat Rev Gastroenterol Hepatol. 2011. https://doi. org/10.1038/nrgastro.2011.131.

7. El-Serag HB. Hepatocellular Carcinoma. N Engl J Med. 2011. https://doi.org/ 10.1056/NEJMra1001683

8. Lubezky N, Facciuto M, Harimoto N, Schwartz ME, Florman SS. Surgical treatment of intrahepatic cholangiocarcinoma in the USA. J Hepatobiliary Pancreat Sci. 2015. https://doi.org/10.1002/jhbp.157.
9. Zhang X, Li J, Shen F, Lau WY. Significance of presence of microvascular invasion in specimens obtained after surgical treatment of hepatocellular carcinoma. J Gastroenterol Hepatol. 2018. https://doi.org/10.1111/jgh.13843.

10. Showe MK, Kossenkov AV, Showe LC. The peripheral immune response and lung cancer prognosis. Oncoimmunology. 2012. https://doi.org/10.4161/ onci.21096.

11. Gustafson MP, Lin Y, LaPlant B, Liwski CJ, Maas ML, League SC, Bauer PR, Abraham RS, Tollefson MK, Kwon ED, Gastineau DA, Dietz AB. Immune monitoring using the predictive power of immune profiles. J Immunother Cancer. 2013. https://doi.org/10.1186/2051-1426-1-7.

12. Buonaguro L, Mauriello A, Cavalluzzo B, Petrizzo A, Tagliamonte M. Immunotherapy in hepatocellular carcinoma. Ann Hepatol. 2019. https://doi. org/10.1016/J.AOHEP.2019.04.003.

13. Connaughton EP, Naicker S, Hanley SA, Slevin SM, Eykelenboom JK, Lowndes NF, O'Brien T, Ceredig R, Griffin MD, Dennedy MC. Phenotypic and functional heterogeneity of human intermediate monocytes based on HLADR expression. Immunol Cell Biol. 2018. https://doi.org/10.1111/imcb.12032.

14. Yu M, Zhou X, Niu L, Lin G, Huang J, Zhou W, Gan H, Wang J, Jiang X, Yin B, Li Z. Targeting transmembrane TNF-a suppresses breast cancer growth. Cancer Res. 2013. https://doi.org/10.1158/0008-5472.CAN-12-3946.

15. Moritz T, Niederle N, Baumann J, May D, Kurschel E, Osieka R, Kempeni J, Schlick E, Schmidt CG. Phase I study of recombinant human tumor necrosis factor alpha in advanced malignant disease. Cancer Immunol Immunother. 1989;29:144-50.

16. Jing Y, Sun K, Liu W, Sheng D, Zhao S, Gao L, Wei L. Tumor necrosis factor-a promotes hepatocellular carcinogenesis through the activation of hepatic progenitor cells. Cancer Lett. 2018. https://doi.org/10.1016/j. canlet.2018.07.001.

17. Gomez D, Farid S, Malik HZ, Young AL, Toogood GJ, Lodge JPA, Prasad KR. Preoperative Neutrophil-to-Lymphocyte Ratio as a Prognostic Predictor after Curative Resection for Hepatocellular Carcinoma. World J Surg. 2008. https://doi.org/10.1007/s00268-008-9552-6.

18. Dan J, Zhang Y, Peng Z, Huang J, Gao H, Xu L, Chen M. Postoperative Neutrophil-to-Lymphocyte Ratio Change Predicts Survival of Patients with Small Hepatocellular Carcinoma Undergoing Radiofrequency Ablation. PLoS ONE. 2013. https://doi.org/10.1371/journal.pone.0058184.

19. Limaye AR, Clark V, Soldevila-Pico C, Morelli G, Suman A, Firpi R, Nelson DR, Cabrera R. Neutrophil-lymphocyte ratio predicts overall and recurrence-free survival after liver transplantation for hepatocellular carcinoma. Hepatol Res. 2013. https://doi.org/10.1111/hepr.12019.

20. Karlmark KR, Weiskirchen R, Zimmermann HW, Gassler N, Ginhoux F, Weber C, Merad M, Luedde T, Trautwein C, Tacke F. Hepatic recruitment of the inflammatory Gr1+ monocyte subset upon liver injury promotes hepatic fibrosis. Hepatology. 2009. https://doi.org/10.1002/hep.22950.

21. Martín-Sierra C, Martins R, Laranjeira P, Abrantes AM, Oliveira RC, Tralhão JG, Botelho MF, Furtado E, Domingues R, Paiva A. Functional impairment of circulating $\mathrm{FccRI}^{+}$monocytes and myeloid dendritic cells in hepatocellular carcinoma and Cholangiocarcinoma patients. Cytom Part B Clin Cytom. 2019. https://doi.org/10.1002/cyto.b.21777.

22. Carlin LM, Stamatiades EG, Auffray C, Hanna RN, Glover G, Vizcay-Barrena G, Hedrick CC, Cook HT, Diebold S, Geissmann F. Nr4a1-dependent Ly6Clow monocytes monitor endothelial cells and orchestrate their disposal. Cell. 2013. https://doi.org/10.1016/j.cell.2013.03.010.

23. Hanna RN, Cekic C, Sag D, Tacke R, Thomas GD, Nowyhed H, Herrley E, Rasquinha N, McArdle S, Wu R, Peluso E, Metzger D, Ichinose H, Shaked I, Chodaczek G, Biswas SK, Hedrick CC. Patrolling monocytes control tumor metastasis to the lung. Science. 2015. https://doi.org/10.1126/science. aac9407.

24. Zimmermann HW, Seidler S, Nattermann J, Gassler N, Hellerbrand C, Zernecke A, Tischendorf JJW, Luedde T, Weiskirchen R, Trautwein C, Tacke F. Functional contribution of elevated circulating and hepatic non-classical CD14CD16 monocytes to inflammation and human liver fibrosis. PLoS One. 2010. https://doi.org/10.1371/journal.pone.0011049.

25. Lopez-Gonzalez JS, Avila-Moreno F, Prado-Garcia H, Aguilar-Cazares D, Mandoki JJ, Meneses-Flores M. Lung carcinomas decrease the number of monocytes/macrophages (CD14+ cells) that produce TNF-a. Clin Immunol. 2007. https://doi.org/10.1016/j.clim.2006.11.003.

26. Verronèse E, Delgado A, Valladeau-Guilemond J, Garin G, Guillemaut S, Tredan O, Ray-Coquard I, Bachelot T, N'Kodia A, Bardin-Dit-Courageot C, Rigal C, Pérol D, Caux C, Ménétrier-Caux C. Immune cell dysfunctions in breast cancer patients detected through whole blood multi-parametric flow 
cytometry assay. Oncoimmunology. 2016. https://doi.org/10.1080/2162402X. 2015.1100791.

27. Geissmann F, Manz MG, Jung S, Sieweke MH, Merad M, Ley K. Development of monocytes, macrophages, and dendritic cells. Science. 2010. https://doi. org/10.1126/science.1178331.

28. Panek CA, Ramos MV, Mejias MP, Abrey-Recalde MJ, Fernandez-Brando RJ, Gori MS, Salamone GV, Palermo MS. Differential expression of the fractalkine chemokine receptor (CX3CR1) in human monocytes during differentiation. Cell Mol Immunol. 2015. https://doi.org/10.1038/cmi.2014.116.

29. Groom JR, Luster AD. CXCR3 ligands: redundant, collaborative and antagonistic functions. Immunol Cell Biol. 2011. https://doi.org/10.1038/ICB. 2010.158 .

30. Liu M, Guo S, Stiles JK. The emerging role of CXCL10 in cancer (review). Oncol Lett. 2011. https://doi.org/10.3892/ol.2011.300.

31. Schutyser E, Struyf S, Van Damme J. The CC chemokine CCL20 and its receptor CCR6. Cytokine Growth Factor Rev. 2003. https://doi.org/10.1016/ S1359-6101(03)00049-2.

32. Chen K-J, Lin S-Z, Zhou L, Xie H-Y, Zhou W-H, Taki-Eldin A, Zheng S-S. Selective recruitment of regulatory $T$ cell through CCR6-CCL20 in hepatocellular carcinoma fosters tumor progression and predicts poor prognosis. PLoS One. 2011. https://doi.org/10.1371/journal.pone.0024671.

33. Liu J, Farmer JD, Lane WS, Friedman J, Weissman I, Schreiber SL. Calcineurin is a common target of cyclophilin-cyclosporin a and FKBP-FK506 complexes. Cell. 1991;66:807-15.

34. Pallet N, Fernández-Ramos AA, Loriot M-A. Impact of immunosuppressive drugs on the metabolism of T cells. Int Rev Cell Mol Biol. 2018. https://doi. org/10.1016/bs.ircmb.2018.05.009.

35. Kannegieter NM, Hesselink DA, Dieterich M, Kraaijeveld R, Rowshani AT, Leenen PJM, Baan CC. The effect of Tacrolimus and Mycophenolic acid on CD14+ monocyte activation and function. PLoS One. 2017. https://doi.org/ 10.1371/journal.pone.0170806.

36. Martín-Sierra C, Martins R, Laranjeira P, Coucelo M, Abrantes AM, Tralhão JG, Botelho MF, Furtado E, Domingues R, Paiva A. Functional and Phenotypic Characterization of Tumor-Infiltrating Leukocyte Subsets and Their Contribution to the Pathogenesis of Hepatocellular Carcinoma and Cholangiocarcinoma. Transl Oncol. 2019. https://doi.org/10.1016/j.tranon. 2019.07.019.

\section{Publisher's Note}

Springer Nature remains neutral with regard to jurisdictional claims in published maps and institutional affiliations.

Ready to submit your research? Choose BMC and benefit from:

- fast, convenient online submission

- thorough peer review by experienced researchers in your field

- rapid publication on acceptance

- support for research data, including large and complex data types

- gold Open Access which fosters wider collaboration and increased citations

- maximum visibility for your research: over $100 \mathrm{M}$ website views per year

At $\mathrm{BMC}$, research is always in progress.

Learn more biomedcentral.com/submissions 